American Journal of Pharmaceutical Education 2020; 84 (9) Article 8046.

\title{
COMMENTARY
}

\section{Positioning the Scholarship of Teaching and Learning Squarely on the Center of the Desk}

\author{
Amy M. Franks, PharmD, ${ }^{\mathrm{a}, \mathrm{b}}$ Nalin Payakachat, $\mathrm{PhD}^{\mathrm{a}}$ \\ ${ }^{\text {a }}$ University of Arkansas for Medical Sciences College of Pharmacy, Little Rock, Arkansas \\ ${ }^{\mathrm{b}}$ Editorial Board Member, American Journal of Pharmaceutical Education, Arlington, Virginia
}

Submitted February 27, 2020; accepted May 20, 2020; published September 2020.

\begin{abstract}
The scholarship of teaching and learning (SOTL) is one of four traditionally defined domains of research. Yet faculty members who pursue the SOTL may feel disadvantaged because of its relatively low perceived value by many institutions. Thus, interested faculty members may relegate the SOTL to a limited, secondary line of research and instead pursue other types of research that appear to be more highly valued by the institution. This commentary explores the benefits to the institution when the SOTL is expressly valued and purposefully supported. We advocate for a multifaceted approach by which pharmacy education leaders can examine how the SOTL is valued, encouraged, and supported through organizational structure and policy. Approaches to solidify and communicate the institutional value of the SOTL are discussed. Specific attention to ensure the SOTL is represented in rewards and incentive systems, such as the college or school's promotion and tenure policies, may empower faculty members to pursue success in this area of research.
\end{abstract}

Keywords: scholarship of teaching and learning, faculty promotion, tenure, institutional culture

\section{INTRODUCTION}

Faculty members have three fundamental responsibilities: teaching, scholarship, and service. They are expected to maintain competence in all three areas within the scope of their individual allocation of effort toward each responsibility. Although policies related to academic promotion vary across institutions, there is a general expectation that faculty members grow in their expertise and excel in at least one of these areas. In the area of scholarship, faculty members are required to stay abreast of emerging knowledge in their area of research so that they can advance the field through well-informed inquiry and disseminated work. This most often applies to more discipline-specific research, and, in many institutions, less emphasis may be placed on faculty members keeping current in the area of teaching and learning. ${ }^{1}$

A decade after Boyer's introduction of the scholarship of teaching, ${ }^{2}$ Shulman refined the definition of the scholarship of teaching and learning (SOTL) to include the critical elements of public availability, susceptibility to peer review, and dissemination to the broader professional community. ${ }^{3}$ The SOTL contributes to the advancement of academic pharmacy, informing teaching

Corresponding Author: Amy M. Franks, University of Arkansas for Medical Sciences College of Pharmacy, 4301 West Markham St., \#522, Little Rock, AR 72205. Tel: 501296-1296. Email: afranks@uams.edu and learning strategies, outcomes assessment, administrative practices, and other educational practices. However, for many faculty members, the SOTL represents an interesting but separate and limited line of scholarship that should not replace their primary research, which often resides within their scientific discipline or some other area of work more traditionally valued by the institution. ${ }^{4-6}$ For example, an assistant professor may be hired in a department of social and administrative sciences (SAS) to fulfill the three academic missions after completing a graduate degree in this field. As this faculty member gains knowledge of and experience in teaching, an SOTL project may naturally develop and result in publication of a peer-reviewed manuscript. Nevertheless, this successful pursuit of the SOTL may be seen as a distraction from work within their specific discipline (ie, SAS), in part because of the vastly different skill sets needed for the two types of research. ${ }^{4,5}$ Further, pursuing the SOTL means choosing not to spend time on other responsibilities because of the limited time and effort available. ${ }^{4}$ Department chairs may be reluctant to allocate faculty time and effort to the SOTL, even if it represents an area of interest and success, since doing so would ultimately detract from other work that may be more highly valued, either formally or informally, by the school or college. ${ }^{5,7}$ For the faculty member, the result is that the SOTL is either abandoned altogether or relegated to an add-on or side 


\section{American Journal of Pharmaceutical Education 2020; 84 (9) Article 8046.}

responsibility. ${ }^{4-6}$ In this example, the interest in the SOTL is pushed to the side of the desk in order for the faculty member to pursue SAS-focused research that is more valued by the institution.

\section{Challenges and Risks of Engaging in SOTL}

Several factors may directly or indirectly impact institutional culture related to the SOTL. First, the SOTL may be viewed as having low yield when measured by traditional scholarly metrics (eg, number of citations, journal impact factor). Traditionally, SOTL publications are less frequently cited than other types of scholarship, and their impact may be more difficult to track with citation metrics. Other ways to measure the impact, such as describing the influence on curricular design or teaching practices at the faculty member's home institution or another institution, may be better markers of the importance of the work. ${ }^{8}$ Second, successful SOTL is disseminated through presentation and publication; however, fewer outlets exist for dissemination of SOTL. Only a few peer-reviewed journals focus content solely on pharmacy education, with the Journal and Currents in Pharmacy Teaching and Learning being most broadly recognized. ${ }^{9}$ Because of the small number of journals and the limited number of articles published per issue in these journals, it may be difficult for well-designed SOTL projects to be disseminated simply because of the competition for publication within these journals.

Challenges associated with the conduct of the SOTL may further dampen institutional enthusiasm and support. All scholarship, including the SOTL, requires faculty time and effort to execute. However, in the authors' experience, little extramural grant funding is directed toward the SOTL compared to other types of research, and when available, the funding amount is often relatively small and rarely supports faculty effort. This lack of salary support can further push SOTL projects to the side of the faculty member's desk. Challenges in the design of SOTL projects may also dissuade faculty from pursuing it without adequate institutional support. Many faculty members, even those with formal research training, need development to effectively design and accomplish SOTL. One faculty member described this new skill development by referring to himself as a "two-sport athlete," noting that his passion for and skills in the SOTL were vastly unlike those for his previous research. ${ }^{4}$ Many SOTL projects take considerable time to complete, especially if multiple student cohorts are needed to study the effects of curricular changes. Comparator groups are difficult to identify, and multicenter investigations are difficult due to substantial inter-institutional differences in curricula. Additionally, faculty members may not know how to effectively design SOTL projects with the safeguards needed to protect students as a vulnerable study population. Institutional support through faculty effort allocation, faculty development programs, and collective expertise would alleviate many of these challenges, but this support simply may not be available for faculty members to utilize.

Faculty members engaged in the SOTL may also face risks related to their career advancement. Smesny and colleagues identified several barriers that detailed why faculty members are conflicted by their interest in the SOTL, including lack of support, funding, and reward/ recognition, and the concern that faculty members pursuing the SOTL are viewed as "second class citizens."10 To sustain their work in the mission areas of scholarship, teaching, and service, faculty members must sense there is institutional value in their work. To ensure both faculty and institutional success, institutions must implement policies and actions that support faculty effort in all types of scholarship. ${ }^{11,12}$ Reward through performance evaluations, promotion and/or tenure, and financial incentives provide faculty members with evidence of the value of their scholarship in the eyes of their institution. Gubbins suggested that institutional values and pride are ultimately reflected in the institution's promotion and tenure policies. ${ }^{11}$ When requirements for scholarship, types of research recognized, and expected deliverables are outlined in promotion and tenure policy and guidance documents, it sends strong signals to faculty members regarding the value attributed to this area. If faculty members do not see the SOTL clearly expressed in the institutional culture and system of values represented in the promotion and tenure policy and guidance documents, they may question its value. They may also abandon the pursuit of SOTL for the sake of career advancement, substituting alternative scholarship perceived to be more valued by the institution. Any resulting pedagogical advances will not be realized, and educational innovation may be diminished. Ultimately, this has a negative effect on the faculty member, their institution, and the Academy as a whole.

\section{Investment in SOTL Benefits Both Faculty and the Institution}

Given the challenges and risks to engaging in the SOTL, why would some faculty members choose to pursue it? Engagement in SOTL provides faculty members with the knowledge, skills, and insight to teach more effectively, and to generate research questions about how their students learn. ${ }^{4,5}$ Devoting time and energy on a skill helps one improve in its practice, and this is no different for teaching. Engagement in the SOTL serves 


\section{American Journal of Pharmaceutical Education 2020; 84 (9) Article 8046.}

as an exciting pathway for faculty development in teaching, encouraging faculty members to perform at the top of their game and subsequently enhancing student learning. ${ }^{4,5,13}$

Further, a productive and robust SOTL program provides the institution with several advantages. In addition to the benefits of the SOTL-engaged faculty members' own development, they also contribute to the greater teaching and learning community by advancing the quality of instruction outside their circle of SOTL scholars. ${ }^{1,13}$ Because these scholars seek awareness and knowledge of emerging data in teaching and learning, they can serve as excellent resources to others across the spectrum of teaching: those interested in enhancing student learning through better teaching, those seeking to adopt sound instructional practices from the literature (scholarly teaching), and those pursuing scholarly work related to their teaching (SOTL). In this sense, the SOTL serves as a mechanism by which to enhance the quality of the institution's educational processes.

Faculty members who are engaged in the SOTL can also more broadly impact the institution by carefully aligning their research with institutional priorities. ${ }^{7}$ This might include initiatives related to the institution's strategic plan, accreditation requirements, or key survey metrics (eg, student or alumni surveys from the American Association of Colleges of Pharmacy [AACP]). ${ }^{7}$ For example, an academic medical center may emphasize interprofessional education in its strategic plan. Faculty members with interest in the SOTL may intentionally seek ways to impact the knowledge, skills, and attitudes toward interprofessional education through their research. Some challenges to the SOTL might be lessened by this approach because of the collective interest and development of support processes, and it might also provide the potential for intramural funding to carry out the work. Faculty members engaged in the SOTL must make the effort to learn about the institution's priorities and look for ways their work can align with these needs. Even in these cases, however, administrators and faculty members leading these institutional priorities must have a keen understanding of SOTL in general and of its practice within the university in order to effectively advocate for its support and inclusion in these discussions. ${ }^{7}$

Finally, the institution's external reputation can be bolstered by the SOTL. A key aim of the SOTL is to enhance student learning, which focuses efforts on the institution's primary external audience: its current and prospective students. ${ }^{1,5,13}$ Just as in any other discipline, as the influence of a faculty member's work grows, the faculty member's reputation also grows. This begins with faculty recognition through the dissemination of their
SOTL through presentations and publications, and may even lead to awards for excellence in teaching and learning. ${ }^{5}$ As a faculty member's reputation grows through more visible dissemination of their research, their contributions are sought even more frequently, resulting in national recognition as an expert in the field. When SOTL-engaged faculty members gain regional or national prominence through the dissemination of their work, the institution can benefit greatly from publicizing these efforts to elevate and advance student learning by their own faculty members. It paints a picture of a vibrant educational community and sends the message that high-quality teaching performance is valued and expected. ${ }^{1,13}$

\section{The Support of the Academy for Recognition of SOTL}

Literature on how institutions can build capacity in the SOTL is limited, but it likely requires support at many levels and attention toward alleviating its challenges. ${ }^{1}$ Smesny and colleagues identified potential solutions to better support SOTL, such as inclusion within promotion and tenure criteria and other reward systems, assurance of protected time, and better education of faculty members and administrators on its value to the institution. ${ }^{10}$ In 2011, Medina and colleagues argued that SOTL should be recognized as an important form of scholarship, deserving of protected time and incentives. ${ }^{14}$ At that time, they observed that only $40(40 \%)$ of 100 schools and colleges of pharmacy included the SOTL in promotion and tenure policy and guidance documents. ${ }^{14}$

Academic pharmacy has called for the SOTL to be recognized as an important and valued form of faculty scholarship. ${ }^{10,14,15}$ In 2017-2018, the AACP prioritized universal recognition of the SOTL and established the Association's SOTL grants. ${ }^{16}$ The first call for proposals was met with enthusiasm, with 90 applications submitted. ${ }^{16}$ In the report of the AACP 2017-2018 Academic Affairs Standing Committee, Fulford and colleagues acknowledged institutional bias against SOTL, stating it is often considered "lesser or secondary" to other scholarship. ${ }^{17}$ The committee strongly advocated for SOTL as an expectation of pharmacy teaching faculty, citing the convergence of many significant drivers of change in teaching and learning in academic pharmacy. ${ }^{17}$ Specifically, they called for pharmacy schools to ensure that promotion and tenure policy and guideline documents identify and assess the SOTL similarly to other types of scholarship, with emphasis on the quality, significance, and impact of the individual's work. ${ }^{17}$

Concerns about the value of SOTL in promotion and tenure decisions are not limited to pharmacy faculty members. ${ }^{6,10,18-20}$ Smesny and colleagues described 


\section{American Journal of Pharmaceutical Education 2020; 84 (9) Article 8046.}

similar barriers related to the lack of recognition and value of the SOTL among medical, dental, nursing, and pharmacy disciplines. ${ }^{10}$ Burns and colleagues surveyed faculty members from two health sciences campuses and found that faculty members agreed that the SOTL is a "real" form of scholarship, but they were neutral about how their university values the SOTL and skeptical of its impact on performance evaluations or promotion and tenure decisions. ${ }^{18}$ Similarly, the majority of dental and dental hygiene faculty members valued SOTL but did not perceive that their institutions highly valued it. ${ }^{20}$ In most research regarding the SOTL, faculty members from a broad range of disciplines felt that SOTL would be an inadequate substitute for research within their given discipline. ${ }^{6,10,18,19}$

\section{Do Colleges and Schools of Pharmacy Really Value SOTL in Today's Academy?}

Nearly a decade after Medina and colleagues reported that only $40 \%$ of schools and colleges of pharmacy include the SOTL in promotion and tenure policy and guidance documents, ${ }^{14}$ we completed a comprehensive systematic review of scholarship criteria within promotion and tenure policy and guidance documents from 121 $(85 \%)$ US-based schools and colleges of pharmacy. We found no change in the use of specific language related to the SOTL, and that it was specifically mentioned in 49 (41\%) schools and colleges' promotion and tenure policy and guidance documents (manuscript under review). Of these, the majority (44 of 49) did not require the SOTL from faculty members, but acknowledged it as one way to provide evidence of advancement criteria. Four schools and colleges documented a qualitative requirement specifically for SOTL. The promotion and tenure policy and guidance document for one of the schools states that "All faculty members at all ranks are expected to demonstrate competency in the scholarship of teaching...the scholarship of teaching may be supported by peer reviewed publications and grants." Another school's promotion and tenure policy and guidance document stated a requirement for "Materials related to teaching performance, including. . .evidence of engagement in the scholarship of teaching and learning," but did not further define how to demonstrate this requirement. The schools and colleges that included the SOTL in their promotion and tenure policy and guidance documents, either as a specific requirement or as supporting evidence, commonly cited presentations, publications, and externally reviewed teaching materials as examples of evidence of the SOTL, thus solidifying acceptance of the critical elements defined by Shulman: public availability, peer review, and dissemination to the broader professional community. ${ }^{3}$
We were discouraged to find that the percentage of schools including the SOTL in promotion and tenure policy and guidance documents has not changed since the 2011 report from Medina and colleagues. ${ }^{14}$ During this time, new schools and colleges of pharmacy opened and recruited faculty members in teaching-intensive roles. According to the steep increase in the number of related search results in Google Scholar during this period, the SOTL advanced markedly between 2011 and 2019 (Figure 1), and we expected a greater percentage of schools and colleges to have included it. Regardless, we applaud the 49 schools and colleges that acknowledged or required the SOTL in their promotion and tenure policy and guidance documents. Promotion and tenure policy and guidance documents represent a roadmap for faculty members to plan and achieve career advancement. As such, it is important that faculty members see their work represented in these documents, ideally with explicit acknowledgment of each domain of scholarship and the associated metrics required to achieve excellence. Through careful reflection and evaluation, faculty members within these 49 schools and colleges should be able to explain how their SOTL work contributes to the institution's collective body of research. However, faculty members within the remaining 72 schools and colleges lack the roadmap needed for advancing their SOTL-focused careers when there is no mention of this type of scholarship in the promotion and tenure policy and tenure documents. These faculty members face a difficult task as they prepare to effectively communicate the importance of their work in the SOTL to their respective promotion and tenure committees when they are uncertain whether their school values this scholarly work.

\section{CONCLUSION}

Academic pharmacy institutions (ultimately, deans, department chairs, and promotion and tenure committee members) must choose whether to intentionally support the pursuit of the SOTL at their schools and colleges. We challenge leaders in pharmacy education to objectively consider whether institutional commitment to the SOTL exists at the school, college, and university levels. Taking the approach suggested by Myatt and colleagues, ${ }^{1}$ we suggest adopting an intentional, multifaceted approach across the various levels of the institution, examining the influence of leadership, policies, and organizational structures on the institutional culture related to the SOTL. Specifically, we call for leaders to examine whether faculty development is needed to help both administrators and faculty better understand the SOTL and its impact. Leaders must advocate for incorporating their faculty members' SOTL data into institutional initiatives, 
A. 6000

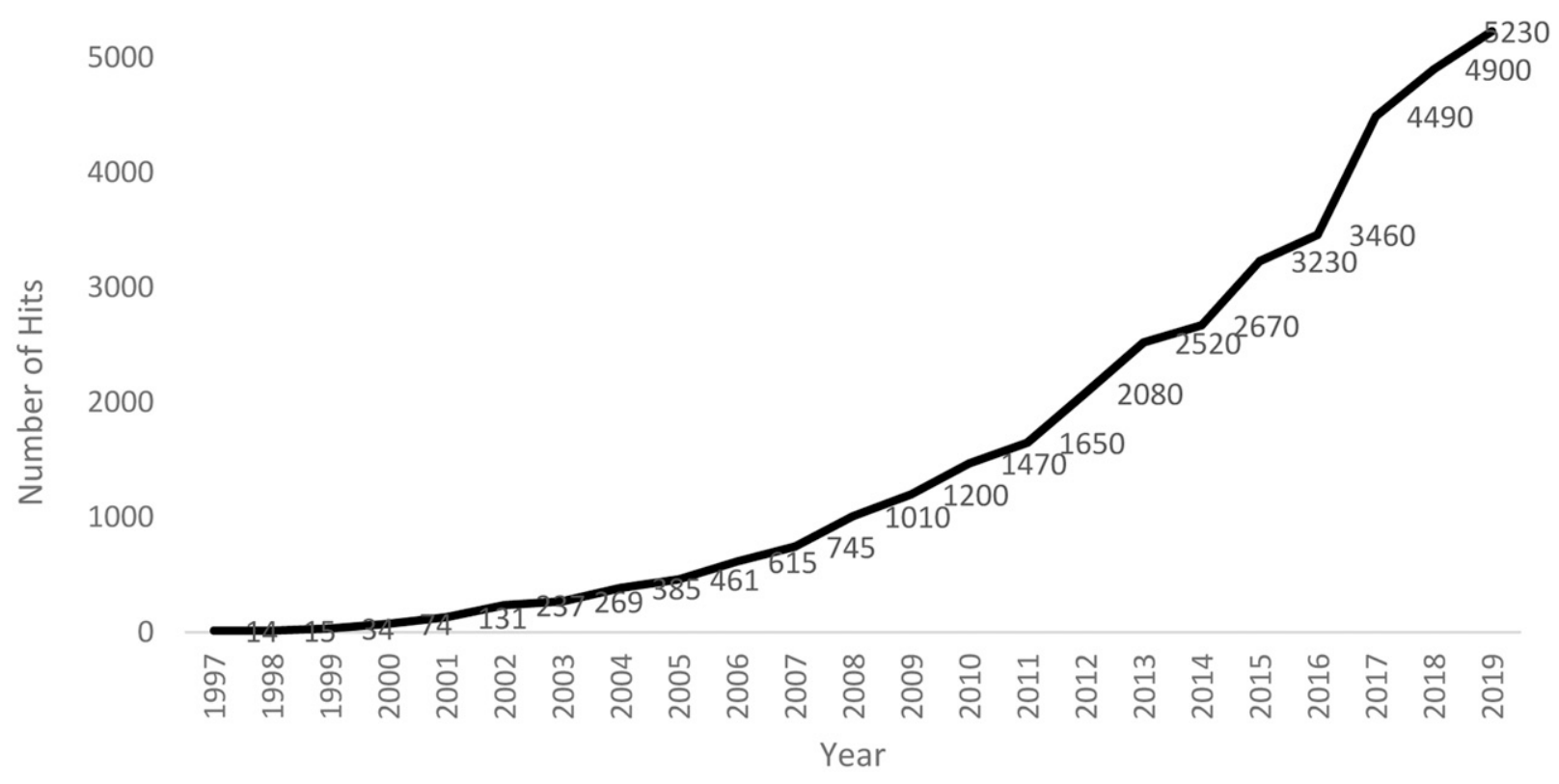

B. 500

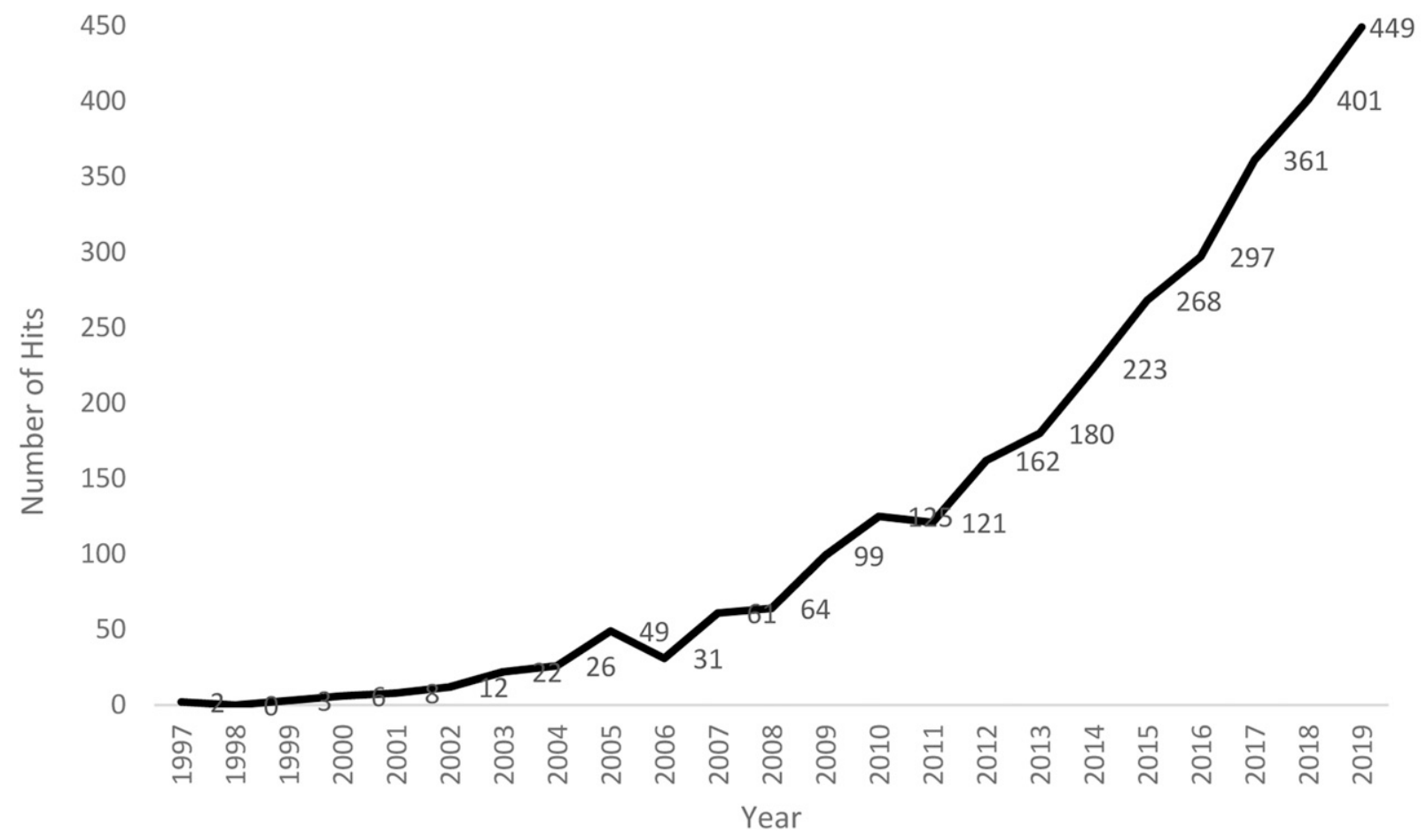

Figure 1. Google Scholar Search Results for Scholarship of Teaching and Learning

Panels A (all results) and B (combined with "pharmacy" search term) show the trend in number of search results over time when using "Scholarship in Teaching and Learning" as the search phrase in Google Scholar. 


\section{American Journal of Pharmaceutical Education 2020; 84 (9) Article 8046.}

helping increase the visibility of the practice of the SOTL as well as the faculty members themselves. We ask these leaders to consider whether the SOTL is included in reward and incentive systems that benefit faculty members engaged in other types of scholarship, and to consider recognition, funding, and effort allocation to support the successful pursuit of the SOTL. Most importantly, we encourage these leaders to reduce ambiguity in promotion and tenure criteria and therefore reduce the individual biases that may exist regarding the value of SOTL as a legitimate scholarly avenue for advancement. A commitment to the SOTL, reflected explicitly in promotion and tenure policy and guidance documents, assures faculty members that their research will be valued and rewarded. Only then should pharmacy faculty members confidently move SOTL-based projects from the side of the desk to squarely in the center of the desk, advancing pharmacy education as a result.

\section{REFERENCES}

1. Myatt P, Gannaway D, Chia I, Fraser K, McDonald J. Reflecting on institutional support for SOTL engagement: developing a conceptual framework. Int J Acad Dev. 2018;23(2):147-160. 2. Boyer EL. Scholarship Reconsidered: Priorities of the Professoriate. Princeton, NJ: Carnegie Foundation for the Advancement of Teaching; 1990.

3. Shulman L. From Minsk to Pinsk: Why a scholarship of teaching and learning? Journal of the Scholarship of Teaching and Learning. 2001:48-53.

4. Draeger J. Why bother with the scholarship of teaching and learning? Insight: A Journal of Scholarly Teaching. 2013;8:12-19. 5. Swart AJ, Luwes N, Olwagen L, Greyling C, Korff C. Scholarship of teaching and learning: 'what the hell' are we getting ourselves into? Eur J Eng Educ. 2017;42(6):653-667.

6. Webb AS. Navigating the lows to gain new heights: constraints to SOTL engagement. The Canadian Journal for the Scholarship of Teaching and Learning. 2019;10(2).

7. Schroeder CM. Countering SOTL marginalization: A model for aligning SOTL with institutional initiatives. International Journal for the Scholarship of Teaching and Learning. 2007;1(1):Article 15.
8. Medina MS, Draugalis JR. Exploring impact metrics beyond indices and citations for SOTL and applied educational research. Am J Pharm Educ. 2019;83(3):7211-7211.

9. Beckett RD, Bicket S, Gill K, et al. AACP core list of journals for pharmacy education, 7th edition, 2019. https://connect.aacp.org/ HigherLogic/System/DownloadDocumentFile.ashx?

DocumentFileKey =881ed081-3442-c8a3-832c-c8203d22802a\& forceDialog=1. Accessed September 2, 2020.

10. Smesny AL, Williams JS, Brazeau GA, Weber RJ, Matthews HW, Das SK. Barriers to scholarship in dentistry, medicine, nursing, and pharmacy practice faculty. Am J Pharm Educ. 2007;71(5):91.

11. Gubbins PO. The scholarship of teaching and learning: an opportunity for clinical faculty members in academic pharmacy and other health professions to develop a program of scholarship.

International Journal for the Scholarship of Teaching and Learning. 2014;8(1):Article 3.

12. Kehrer JP, Svensson CK. Advancing pharmacist scholarship and research within academic pharmacy. Am J Pharm Educ.

2012;76(10):Article 187.

13. Bernstein D. How SOTL-active faculty members can be cosmopolitan assets to an institution. Teaching \& Learning Inquiry: The ISSOTL Journal. 2013;1(1):35-40.

14. Medina M, Hammer D, Rose R, et al. Demonstrating excellence in pharmacy teaching through scholarship. Curr Pharm Teach Learn. 2011;3(4):255-259.

15. Kennedy DR, Calinski DM. P\&T and Me. Am J Pharm Educ. 2018;82(8):Article 7048.

16. Scott S. Address of the 2017-2018 President to the Opening General Session at the 2018 Annual Meeting. Am J Pharm Educ. 2019;83(3):7594.

17. Fulford MJ, Early JL, Godwin DA, et al. Report of the 2017-2018 Academic Affairs Standing Committee. Am J Pharm Educ. 2018;82(7):7163.

18. Burns S, Merchant C, Appelt E. Campus survey on the status of the scholarship of teaching and learning (SoTL) by health sciences faculty. Education. 2013;133(4):502-505-512.

19. Hutchings P, Huber M, Ciccone A. Getting there: an integrative vision of the scholarship of teaching and learning. International Journal for the Scholarship of Teaching and Learning. 2011;5:Article 31.

20. Lanning SK, McGregor M, Crain G, Van Ness CJ, Keselyak NT, Killip JW. The status of the scholarship of teaching and learning in dental education. J Dent Educ. 2014;78(10):1353-1363. 